\title{
Description Logic Concept
}

National Cancer Institute

\section{Source}

National Cancer Institute. Description Logic Concept. NCI Thesaurus. Code C42717.

A terminology concept modeled in a structured and formally well-understood way using logic-based semantics which can be given by a translation into first-order predicate logic. 